\title{
Maintaining Weight and Nutritional Status with Ninjin'yoeito in Elderly Patients with Chronic Wasting Diseases
}

Yumi Nakagawa, ${ }^{1}$ Katsunori Kagohashi, ${ }^{2}$ Ayako Shioya, ${ }^{3}$ Kensuke Kinoshita, ${ }^{4}$ Hiroshi Tsuji, ${ }^{5}$ Hiroaki Satoh ${ }^{2}$

ABSTRACT: Objectives: This retrospective study aimed to obtain information on the nutritional maintenance effects of Ninjin'yoeito (NYT) in elderly patients with chronic wasting diseases. Methods: Changes in body weight and serum levels of total protein and albumin were investigated in patients who received NYT for chronic wasting diseases for more than six months in Mito Medical Centre, University of Tsukuba-Mito Kyodo General Hospital, Mito, Japan, from April 2009 to October 2019. Results: During the study period, 11 patients (median age: 75 years) received NYT for six months or more. The median administration period of NYT was 14 months. The body weight and serum levels of total protein and albumin at the time of the last observation were not significantly different from those at the beginning of NYT administration $(P=0.176, P=0.766$ and $P=0.550$, respectively). Conclusion: This study suggested the possibility of maintaining nutritional condition by administering NYT in elderly patients with chronic wasting disease of various aetiologies. More evidence will be required to confirm these results.

Keywords: Body Weight; Nutritional Status; Ninjin'yoeito; Aged; Chronic Wasting Disease.

$\mathrm{S}$ EVERAL KAMPO MEDICINES (JAPANESE TRADItional herbal medicines) have shown favourable nutritional effects in patients with chronic wasting diseases. ${ }^{1}$ Among kampo medicines, hozai is a term used to refer to any drug that supplements what one is lacking physically and mentally. ${ }^{1}$ Ninjin'yoeito (NYT) is a type of hozai that has been used for improving disturbances and imbalances in the homeostatic condition of the body., This kampo medicine is comprised of 12 herbs, including ginseng, one of the most famous herbal medicines used to treat chronic wasting disease symptoms. ${ }^{4}$ It is hypothesised that NYT might be a candidate drug for the treatment of frailty symptoms observed in the elderly. ${ }^{5}$

A retrospective study was conducted to obtain information on the nutritional maintenance effects of NYT in elderly patients with chronic wasting diseases.

\section{Methods}

Medical records of all of the patients who received NYT for chronic wasting diseases for more than six months in Mito Medical Centre, University of Tsukuba-Mito Kyodo General Hospital, Mito, Japan, from April 2009 to October 2019 were reviewed. Body weight and serum levels of total protein and albumin in these patients were evaluated at the beginning and end of NYT administration. A $2.5 \mathrm{~g}$ or $3 \mathrm{~g}$ (depending on the manufacturing company) dosage of NYT was orally administered three times per day. To rule out its possible effects on body weight and serum levels of protein and albumin, patients were treated with continuous administration of corticosteroids and those with serum protein transfusion during the study period were excluded from this study.

Due to the small number of patients who took NYT for a long period, instead of carrying out a background-matched property case-control study, comparisons of body weight and serum levels of total protein and albumin before and after NYT administration were performed for each patient in this study. Data were analysed using statistical package for social sciences (SPSS), version 23 (IBM Corporation, Armonk, New York). The Wilcoxon rank-sum test, a non-parametric test of paired samples, was used to compare the samples.

Follow-up sessions were conducted with patients for more than six months and it was confirmed that no patients received drugs that might affect the nutrition of the patients after the start of NYT administration.

This study was approved by the Institutional Review Board of Mito Medical Centre, University of Tsukuba-Mito Kyodo General Hospital (Nos. 1526). Comprehensive written informed consent was obtained from each patient.

\section{Results}

For the study period, 11 patients (males $=6$, females $=5$ ) who received NYT for six months or more were found in the in-hospital database. The median age was 75 years (56-90 years). All 11 patients had lost 
their appetite and eight of them had general fatigue. The sample's underlying chronic diseases were as follows: four patients had respiratory diseases (two patients with chronic obstructive pulmonary disease, one with nontuberculous mycobacteriosis and one with combined pulmonary fibrosis and emphysema), four had neurological diseases (two patients with Parkinson's disease and two with polyneuropathy) and three had digestive diseases (two patients with chronic gastritis and one with gastric ulcer). The median administration period of NYT was 14 months (8-61 months). The participants' body weight at the last observation (median: $47.9 \mathrm{~kg}$, range: $43.5-70.0 \mathrm{~kg}$ ) was not significantly different from their body weight at the start of administration (median: $48 \mathrm{~kg}$, range: 42.7-70.0 kg; $P=0.176$ ). Moreover, there was no statistically significant difference in serum total protein level (median: $7.0 \mathrm{~g} / \mathrm{dL}$, range: $5.7-8.2 \mathrm{~g} / \mathrm{dL}$ versus median: $6.9 \mathrm{~g} / \mathrm{dL}$, range: $5.8-7.9 \mathrm{~g} / \mathrm{dL} ; P=0.766$ ). Their serum albumin level at the last observation (median: $3.9 \mathrm{~g} / \mathrm{dL}$, range: $2.8-4.6 \mathrm{~g} / \mathrm{dL}$ ) was not lower than that at the start of administration (median: $3.9 \mathrm{~g} /$ $\mathrm{dL}$, range: $2.4-4.6 \mathrm{~g} / \mathrm{dL} ; P=0.550$ ). Among the male patients, changes in body weight and serum levels of total protein and albumin between the start and end of NYT administration were not statistically significant $(P=0.276)$. There were no changes in these three indicators between the two measurements in time $(P$ $=0.285$ ) among the female patients either.

\section{Discussion}

Since the $18^{\text {th }}$ century, NYT has been prescribed in Japan according to the original text (Wazaikyokuho, Héjì Júfāng in Chinese: a collection of prescriptions for medicines published in China in the $12^{\text {th }}$ century) without any changes. ${ }^{6} \mathrm{NYT}$ is made up of a combination of several crude herbal medicines: rehmannia glutinosa root, Japanese angelica root, atractylodes lancea, poria sclerotium, ginseng, cinnamon bark, polygala root, peony root, citrus unshiu peel, astragalus root, glycyrrhiza and schisandra fruit. ${ }^{6}$ Of these herbal medicines, ginseng is one of the most famous herbal medicines and has been administered to patients with chronic wasting diseases. ${ }^{4} \mathrm{NYT}$ has been reported to be effective in treating anorexia in elderly individuals and fatigue in patients with cancer in various organs. ${ }^{2,5}$ The effects of NYT on frailty, cachexia and sarcopenia in patients with malignant and non-malignant diseases have been studied. ${ }^{2,5}$ However, in English literature, only a few studies have reported the long-term effects of this kampo medicine on body weight gain in patients with various chronic wasting diseases. ${ }^{2,5}$ Given this background, this study sought to examine the effects of NYT on the maintenance of body weight and nutritional status in elderly patients with chronic wasting diseases.

Recently, Suzuki et al. conducted a postmarketing survey among elderly patients to evaluate the safety and effectiveness of NYT. ${ }^{5}$ In this survey, the safety and effectiveness of NYT in 808 elderly patients were evaluated. The median age was 78 years and body weights at both baseline and week 24 were analysed in 275 (34.0\%) of 808 patients. It was reported that the mean body weight in these 275 patients increased significantly from $49.4 \pm 9.0 \mathrm{~kg}$ at baseline to $50.0 \pm 9.0 \mathrm{~kg}$ at week $24(P<0.01)$. There was a significant difference in women $(P<0.01)$, but not in men. Although body weight increased by $0.6 \mathrm{~kg}$ on average, the increment did not apply to all the 808 patients, and body weight was analysed using a paired $\mathrm{t}$-test, which is not a non-parametric test. In addition, serum levels of total protein and albumin were not evaluated in that study. ${ }^{5}$ It was interesting that the mean or median body weight was around $50 \mathrm{~kg}$ in the post-marketing survey as well as in the current study. The differences in changes of body weight in the postmarketing survey and this study could be attributed to the difference in background diseases and the method considered for statistical analysis .

A characteristic of the kampo medicinal approach is to deal with anorexia due to different pathologies in an integrated manner, rather than by engaging in comparative analysis using more Western medical means. Therefore, it would be meaningful to disseminate the information on the treatment results for patients who wish to be treated with herbal medicine.

Although this is a small-sized study without a comparative group and could not be comprehensive enough to draw generalisable conclusions, the information obtained from this study could help future researchers explore the role of NYT in treatments as an alternative to otherwise mainstream Western avenues.

\section{Conclusion}

The results obtained in this study suggested the possibility of maintaining nutritional condition by administration of NYT in elderly patients with chronic wasting disease of various aetiologies. As there was no control group in this study, future studies should include a control group to enable a solid conclusion to be drawn. 


\section{CONFLICTS OF INTEREST}

The authors declare no conflicts of interest.

\section{FUNDING}

No funding was received for this pilot study.

\section{AUTHORS' CONTRIBUTION}

YN, KaK and HS designed the study. YN, KaK, AS, KeK, HT and HS collected the data. YN, KaK and HS analysed the data and prepared the manuscript. All authors approved the final version of the article.

\section{References}

1. Amitani M, Amitani H, Sloan RA, Suzuki H, Sameshima N, Asakawa A, et al. The translational aspect of complementary and alternative medicine for cancer with particular emphasis on Kampo. Front Pharmacol 2015; 6:150. https://doi.org/10.3389/ fphar.2015.00150.
2. Miyano K, Nonaka M, Uzu M, Ohshima K, Uezono Y. Multifunctional actions of Ninjinyoeito, a Japanese Kampo medicine: Accumulated scientific evidence based on experiments with cells and animal models, and clinical studies. Front Nutr 2018; 5:93. https://doi.org/10.3389/fnut.2018.00093.

3. Scheid V, Bensky D, Ellis A, Barolet R. Chinese Herbal Medicine Formulas and Strategies. Seattle, USA: Eastland Press, Inc., 2009. Pp. 505-618.

4. Shergis JL, Di YM, Zhang AL, Vlahos R, Helliwell R, Ye JM, et al. Therapeutic potential of Panax ginseng and ginsenosides in the treatment of chronic obstructive pulmonary disease. Complement Ther Med 2014; 22:944-53. https://doi.org/10.10 16/j.ctim.2014.08.006.

5. Suzuki S, Aihara F, Shibahara M, Sakai K. Safety and effectiveness of Ninjin'yoeito: A utilization study in elderly patients. Front Nutr 2019; 6:14. https://doi.org/10.3389/fnut.2019.00014.

6. Terasawa, K. Japanese and Chinese Medical Practice Learned from Cases (in Japanese). Tokyo, Japan: Igaku-Shoin Ltd., 1990. 